\title{
Oleic Acid in the Ventral Tegmental Area Inhibits Feeding, Food Reward, and Dopamine Tone
}

\author{
Cecile Hryhorczuk ${ }^{1,2}$, Zhenyu Sheng ${ }^{3}$, Léa Décarie-Spain ${ }^{1,4}$, Nicolas Giguère ${ }^{5}$, Charles Ducrot ${ }^{5}$, \\ Louis-Éric Trudeau ${ }^{5}$, Vanessa H Routh ${ }^{3}$, Thierry Alquier ${ }^{1,6}$ and Stephanie Fulton, ${ }^{* 1,7}$ \\ 'CRCHUM and Montreal Diabetes Research Center, Montréal, QC, Canada; '2Department of Physiology, Université de Montréal, Montréal, QC, \\ Canada; ${ }^{3}$ Rutgers New Jersey Medical School, Department of Pharmacology, Physiology and Neuroscience, Rutgers University, Newark, NJ, USA; \\ ${ }^{4}$ Department of Neurosciences, Université de Montréal, Montréal, QC, Canada; ${ }^{5}$ Department of Pharmacology, Université de Montréal, Montréal, \\ QC, Canada; 'Department of Medicine, Université de Montréal, Montréal, QC, Canada; 'Department of Nutrition, Université de Montréal, \\ Montréal, QC, Canada
}

\begin{abstract}
Long-chain fatty acids (FAs) act centrally to decrease food intake and hepatic glucose production and alter hypothalamic neuronal activity in a manner that depends on FA type and cellular transport proteins. However, it is not known whether FAs are sensed by ventral tegmental area (VTA) dopamine (DA) neurons to control food-motivated behavior and DA neurotransmission. We investigated the impact of the monounsaturated FA oleate in the VTA on feeding, locomotion, food reward, and DA neuronal activity and DA neuron expression of FA-handling proteins and FA uptake. A single intra-VTA injection of oleate, but not of the saturated FA palmitate, decreased food intake and increased locomotor activity. Furthermore, intra-VTA oleate blunted the rewarding effects of high-fat/sugar food in an operant task and inhibited DA neuronal firing. Using sorted DA neuron preparations from TH-eGFP mice we found that DA neurons express FA transporter and binding proteins, and are capable of intracellular transport of long-chain FA. Finally, we demonstrate that a transporter blocker attenuates FA uptake into DA neurons and blocks the effects of intra-VTA oleate to decrease food-seeking and DA neuronal activity. Together, these results suggest that DA neurons detect FA and that oleate has actions in the VTA to suppress DA neuronal activity and food seeking following cellular incorporation. These findings highlight the capacity of DA neurons to act as metabolic sensors by responding not only to hormones but also to FA nutrient signals to modulate food-directed behavior.
\end{abstract}

Neuropsychopharmacology (2018) 43, 607-6I6; doi:I0.1038/npp.2017.203; published online 27 September 2017

\section{INTRODUCTION}

Long-chain fatty acids (FAs) are among the metabolic signals that are centrally detected to control energy homeostasis. Initial work discovered that hypothalamic neurons alter their firing rate in response to FA application (Oomura et al, 1975) while more recent studies demonstrated the impact of centrally administered FA to suppress feeding and hepatic glucose production (Lam et al, 2005; Obici et al, 2002; Schwinkendorf et al, 2011). The ability of FA to inhibit food intake and modulate autonomic function and neurotransmission is reported to be mediated by cellular FA transport and intracellular metabolism (Lam et al, 2005; Le Foll et al, 2013; Moulle et al, 2013; Obici et al, 2003). In turn, blocking FA hydrolysis from triacylglycerol in the brain has been shown to increase appetite and weight gain (Cansell et al, 2014; Picard et al, 2014b).

*Correspondence: Professor S Fulton, CRCHUM and Montreal Diabetes Research Center, 900 rue Saint-Denis, Office 8-428, Montreal, QC, Canada H2X OA9, Tel: +5/4.890.8000 × 23602, E-mail: stephanie.fulton@umontreal.ca

Received 2 February 2017; revised 23 August 2017; accepted 28 August 2017; accepted article preview online 31 August 2017
Several lines of evidence indicate that the influence of FA on energy metabolism depends on the chemical structure of the FA chain. The monounsaturated FA oleate $(\mathrm{OA})$ and the saturated FA palmitate (PA) are the most abundant longchain FAs in circulation and can exert different signaling and metabolic actions in the brain. OA, the most extensively produced FA in nature, has been shown to decrease food intake when acutely administered into the third ventricle (Obici et al, 2002; Schwinkendorf et al, 2011) and to inhibit or excite hypothalamic neurons (Jo et al, 2009; Le Foll et al, 2009; Wang et al, 2006). In addition, we have shown that OA has a distinct intracellular metabolic fate in neural cells as compared to PA (Taib et al, 2013).

The mesolimbic dopamine (DA) system has a key role in mobilizing behavior required to meet energy demands. DA neurons of the ventral tegmental area (VTA) respond to numerous hormones and peptides to modulate DA tone and food-directed behaviors (Fulton, 2010; Liu and Borgland, 2015). Prolonged intake of high-fat diets and the development of obesity can elicit molecular and neural adaptations in DA signaling that are associated with blunted reward responses and enhanced food seeking (Davis et al, 2008; Décarie-Spain et al, 2016; Fulton et al, 2006; Hryhorczuk et al, 2015; Johnson and Kenny, 2010; Stice et al, 2008; Wang 
et al, 2001). We recently demonstrated that long-term intake of dietary lipids enriched in PA (from palm oil) dampens mesolimbic DA function while a high-fat diet containing mostly OA (olive oil) is protective against such changes (Hryhorczuk et al, 2015). While these results suggest that the effects of chronic high-fat feeding on DA tone rely on the class of dietary lipids, it is not known whether FAs are detected by DA neurons and whether they can modulate appetite and mesolimbic DA neurotransmission. Here we report that intra-VTA injection of OA, but not PA, inhibited free-feeding intake and stimulated locomotor activity. VTA $\mathrm{OA}$ also blunted the rewarding effects of sucrose in an operant task and decreased DA neuronal firing amplitude and frequency of excitatory inputs onto DA neurons. DA neurons isolated from TH-eGFP mice were found to express genes encoding FA-handling proteins and we provide evidence for cellular transport of long-chain FA in DA neurons using a fluorescent FA analog. Finally, we demonstrate that a transporter blocker not only attenuates FA uptake into DA neurons but also blocks the effects of intra-VTA OA to suppress food-motivated responding and DA neuronal activity. Together, these findings suggest that FAs are detected by DA neurons and that the monounsaturated FA OA can suppress DA neuronal activity and the rewarding effects of food following cellular incorporation.

\section{MATERIALS AND METHODS}

\section{Animals}

Experiments were approved by the CRCHUM Animal Care Committee, the Université de Montréal Animal Ethics Committee, and the Institutional Animal Care and Use Committee of the New Jersey Medical School. All behavioral testing was carried out in rats to maintain precise VTA micro-injections, whereas remaining experiments were performed in mice due to the availability of transgenic lines and methods established for primary DA neuron cultures. Male Wistar rats (Charles River, Saint-Constant, Quebec) weighing 250-280 g upon arrival were housed in reversecycle rooms (lights off at 1000 hours) for 8-10 days before surgery. Male C57Bl/6 mice (4-6 weeks old) were used for electrophysiological recordings. TH-eGFP transgenic mice (C57Bl/6 background) expressing green fluorescent protein (GFP) under the control of the tyrosine hydroxylase (TH) promoter (Matsushita et al, 2002) were used for fluorescence-activated cell sorting (FACS). Primary DA neuron cultures were derived from postnatal (P0-P2) C57Bl/6 mice (Fasano et al, 2008).

\section{Intra-VTA Injections}

Stereotaxic surgery was carried out under isoflurane anesthesia and guided by rat brain atlas coordinates (Paxinos and Watson, 1998). Rats were implanted with bilateral guide cannula (26 gauge, Plastics One) terminating $2 \mathrm{~mm}$ above the VTA $(5.8 \mathrm{~mm}$ posterior to the bregma, $\pm 0.6 \mathrm{~mm}$ from midline, and $-6 \mathrm{~mm}$ from dura). OA and PA $\left(\mathrm{Na}^{+}\right.$; Sigma-Aldrich) were complexed to hydroxypropyl- $\beta$ cyclodextrin (HPB, CTD Holdings, Alachua, FL, USA) and dissolved in artificial cerebrospinal fluid (aCSF, in mM: 125 $\mathrm{NaCl}, 2.5 \mathrm{KCl}, 0.3 \mathrm{KH}_{2} \mathrm{PO}_{4}, 26 \mathrm{NaHCO}_{3}, 10$ glucose, 1.3
$\mathrm{MgSO}_{4}$, and $2.4 \mathrm{CaCl}_{2} ; \mathrm{pH} 7.4$ ) to a final amount of $6 \mathrm{nmol}$ per side. The $6 \mathrm{nmol}$ dose represents $1 / 5$ of that used in prior studies assessing feeding in response to third ventricle $\mathrm{OA}$ and PA complexed to HPB (30 nmol-Obici et al, 2002; Schwinkendorf et al, 2011). As we found a significant anorectic response to $60 \mathrm{nmol}$, but not $30 \mathrm{nmol}$, of OA infused into the lateral ventricle (data not shown) we used a 10:1 ventricle to parenchyma dilution to arrive at the $6 \mathrm{nmol}$ intra-VTA dose. Phloretin (EMD Millipore) was solubilized in $15 \%$ ethanol and diluted to $100 \mu \mathrm{M}$ in aCSF. Substances were delivered in a volume of $500 \mathrm{nl}$ per side at a rate of $100 \mathrm{nl} / \mathrm{min}$ using a bilateral internal cannula (Plastics One) that projected $2 \mathrm{~mm}$ beyond the guide cannula.

\section{Food Intake}

Two cohorts of rats (total $n=41$ ) were used to evaluate the effect of intra-VTA OA and PA on chow intake. Rats were habituated to powder chow diet for 4-7 days. Food was removed $3 \mathrm{~h}$ before injection of $\mathrm{OA}$ or vehicle, or $\mathrm{PA}$ or vehicle, which occurred immediately preceding the onset of the dark cycle. Food cups filled with powder chow were fastened inside a larger cup to catch spillage. Food consumption and body weight were monitored at 2, 6, 12, and $21 \mathrm{~h}$ post injection.

\section{Locomotor Activity}

Ambulatory activity was evaluated in metabolic chambers (AccuScan Instruments, Columbus, OH, USA) in which rats were habituated $24 \mathrm{~h}$ before FA injection. Injections were done just before the onset of the dark phase.

\section{Operant Responding}

Another group of rats $(n=10)$ with bilateral VTA cannulae were trained to respond for $45 \mathrm{mg}$ high-fat plus high-sucrose pellets (TestDiet, Saint Louis, MO, USA) on a progressive ratio schedule of reinforcement in rat operant chambers with retractable levers (Med Associates, St. Albans, VT, USA) as described previously (Sharma et al, 2012). Once stable breakpoint responding was achieved, the effects of an intraVTA vehicle injection was assessed followed by an injection of OA the following day. After breakpoint responding restabilized (number of rewards earned did not vary by more than 15\%), operant responding was assessed in the same animals in response to vehicle and then $\mathrm{OA}+$ phloretin ( $100 \mu \mathrm{M}$ in $0.5 \%$ ethanol) co-injection. Behavioral testing began $1 \mathrm{~h}$ after injection at the beginning of the dark phase. Cannula placement was verified by injections of methylene blue (500 $\mathrm{nl}$ per side) after completion of the experiment.

\section{Electrophysiology}

Whole-cell patch-clamp recordings were performed as described previously (Sheng et al, 2014). Briefly, $300 \mu \mathrm{m}$ coronal slices containing the VTA were cut with a vibratome and maintained at room temperature for at least $1 \mathrm{~h}$ in oxygenated aCSF (in mM: $126 \mathrm{NaCl}, 1.9 \mathrm{KCl}, 1.2 \mathrm{KH}_{2} \mathrm{PO}_{4}$, $26 \mathrm{NaHCO}_{3}, 2.5$ glucose, $1.3 \mathrm{MgCl}_{2}$, and $2.4 \mathrm{CaCl}_{2} ; \mathrm{pH} 7.4$ ) before being transferred to the recording chamber. DA neurons were identified by the presence of an H current $\left(I_{h}\right)$. 
Only cells with membrane potentials more negative than $-45 \mathrm{mV}$ in $2.5 \mathrm{mM}$ glucose and with action potentials that overshoot $0 \mathrm{mV}$ were used for recording. Only cells with an access resistance under $30 \mathrm{M} \Omega$ that did not vary by more than $20 \%$ throughout the experiment were kept for analysis. A total of 29 neurons were analyzed. Borosilicate pipettes (4$6 \mathrm{M} \Omega$; Sutter Instruments, Novato, CA) were filled with an intracellular solution containing (in $\mathrm{mM}$ ): $128 \mathrm{~K}$-gluconate, $10 \mathrm{KCl}, 4 \mathrm{KOH}, 10 \mathrm{HEPES}, 4 \mathrm{MgCl}_{2}, 0.5 \mathrm{CaCl}_{2}, 5 \mathrm{EGTA}, 2$ $\mathrm{Na}_{2} \mathrm{ATP}$, and $0.4 \mathrm{Na}_{2} \mathrm{GTP} ; \mathrm{pH}$ 7.2. Cells with membrane potentials more negative than $-45 \mathrm{mV}$ in $2.5 \mathrm{mM}$ glucose and action potentials, which overshoot $0 \mathrm{mV}$ were considered viable for recording. Miniature excitatory postsynaptic currents (mEPSCs) were recorded in neurons voltageclamped at $-60 \mathrm{mV}$ in extracellular solution supplemented with $1 \mu \mathrm{M}$ tetrodotoxin (TTX, to block presynaptic action potentials) and $20 \mu \mathrm{M}$ bicuculline (to block $\mathrm{GABA}_{\mathrm{A}}$ currents). A unit of $6 \mu \mathrm{M}$ OA was bath perfused for $10 \mathrm{~min}$. In the transporter blockade condition, $100 \mu \mathrm{M}$ phloretin was co-applied with $\mathrm{OA}$ for an additional $10 \mathrm{~min}$. Recordings from the last minute of each condition were used in the analysis.

\section{Fluorescence-Activated Cell Sorting}

P0-P2 mice were cryo-anesthetized and decapitated for tissue collection. As described previously (Fulton et al, 2011; Mendez et al, 2008), freshly dissociated cells from the VTA were prepared and GFP-positive neurons were purified by FACS and directly collected in Trizol (Qiagen, Toronto, ON, Canada).

\section{Quantitative PCR}

Total RNA from VTA and ventromedial hypothalamus (VMH) microdissections and FACS-sorted cells were extracted using Trizol. Concentration and purity of RNA were systematically assessed. RNA were reverse-transcribed into cDNA using the Moloney Murine Leukemia Virus Reverse Transcriptase (M-MLV RT, Life Technologies, Burlington, ON, USA). Genes were amplified using the Rotor-Gene SYBR Green PCR Kit (Qiagen) in the presence of appropriate primer pairs. Results were normalized to $\beta$-actin or cyclophilin and analyzed using the standard curve method (Taib et al, 2013).

\section{Primers}

Primers were designed using BLAST ( U.S. National Library of Medicine) and synthesized by Integrated DNA Technologies based on the following sequences: $a c t b$ ( $\beta$-actin) forward: 5-'TTCTTGGGTATGGAATCCTGTGGCA-3', reverse: 5'-ACCAGACAGCACTGTGTTGGCATA-3'; $c d 36$ forward: 5'-TGC ATGAATTAGTTGAACCAGGCCA-3', reverse: CCACAGTT CCGATCGCAGCC-3', ppia (cyclophilin) forward: 5'-GCTTTT CGCCGCTTGCTGCA-3', reverse: TGCAAACAGCTCGAAGGAGACGC-3'; fabp3 forward: 5'-GATGACCGGAAGGTCAAGTC-3', reverse: 5'-GCCATGAGTGAGAGTCAGGA-3'; fabp5 forward: 5'-AAACCGAGAGCACAGTGAAGA-3', reverse: 5'-AAGGTGCAGACCGTCTCAGT-3'; fabp7 forward: 5'-AGTACATGAAGGCTCTGGGCGTG-3', reverse: 5'-ATCA CCACTTTGCCGCCTTCCT-3'; fatp1 forward: 5'-GCAG
GTACTACCGCATTGCT-3', reverse: 5'-GAACTTCTTGCGC AGTACCA-3'; fatp4 forward: 5'-TGTGGTGCACAGCAGGTA TT-3', reverse: 5'-TTTCCTGCTGAGTGGTAGAGG-3'; gad1 (GAD67) forward: 5'-ATATCATTGGTTTAGCTGGTGA ATG-3', reverse: 5'-GTGACTGTGTTCTGAGGTGAAGAG-3'; th forward: 5'-CGACCCGTGGCCGGTCTAC-3', reverse: 5'GCAGTCTGGCTCGGGTGAGTG-3'.

\section{FA Uptake in DA Neurons}

Midbrain DA neurons grown on a cortical glial layer were prepared from P0-P2 wild-type mice as described (Fasano et al, 2008). FA uptake was assessed with C1-BODIPY 500/510-C12, a fluorescent FA analog that with the fluorophore head resembles the chemical structure of a long-chain FA (20 $\mu \mathrm{M}$ in $0.8 \%$ DMSO; ThermoFisher). Ten days after seeding, cells were treated with BODIPY, BODIPY + phloretin $(100 \mu \mathrm{M}$ in $0.5 \%$ ethanol), or vehicle alone in culture medium (Neurobasal additioned with glutamax) for 45 min. Following three washes with ice-cold PBS cells were fixed with $10 \%$ formalin for $10 \mathrm{~min}$. TH immunofluorescence was carried out as described previously (Fulton et al, 2011). The ImageJ analyzing tool (http://imagej.nih.gov/ij/) was used to determine BODIPY fluorescence intensity. For each condition, integrated density was measured in $\times 63$ magnified fields $(n=45)$ derived from three individual coverslips per condition.

\section{Statistical Analyses}

Data are presented as mean \pm SEM and were analyzed using GraphPad Prism (version 5.02 for Windows GraphPad Software, San Diego, CA, USA). Two-way analysis of variance (ANOVA) with Bonferonni post-tests was used to analyze food intake and locomotor activity data. One-way ANOVA was used in electrophysiology and immunofluorescence with Bonferonni post-tests. Operant responding and quantitative PCR (qPCR) data were analyzed with Student's $t$-test. Statistical significance was set at $p \leqslant 0.05$.

\section{RESULTS}

\section{Intra-VTA Oleate Inhibits Food Intake and Stimulates Locomotor Activity}

We tested the acute effects of intra-VTA OA and PA on food intake. As illustrated in Figure $1 \mathrm{a}$ and $\mathrm{b}$, OA significantly inhibited chow intake $12 \mathrm{~h}$ after injection (vehicle: $23.9 \pm 1.3 \mathrm{~g}$, OA: $19.6 \pm 1.6 \mathrm{~g} ; 18 \%$ decrease; repeated measures two-way ANOVA; $\mathrm{F}_{1,21}=4.47, p<0.05$ ) while stimulating ambulatory locomotor activity $\left(\mathrm{F}_{1,12}=5.55, p<0.05\right)$. Comparatively, an equimolar dose of $\mathrm{PA}$ in a different cohort of animals did not alter chow intake $\left(\mathrm{F}_{1,14}=0.46, p>0.05\right)$ or locomotion $\left(\mathrm{F}_{1,16}=0.07, p>0.05\right.$; Figure $1 \mathrm{c}$ and $\left.\mathrm{d}\right)$. Bilateral VTA cannula coordinates were confirmed by rhodamine microbead injections (Supplementary Figure S1A). The anorectic effects of OA or PA were not associated with local inflammation as IL- $1 \beta$ and TNF $\alpha$ mRNA levels in the VTA were unchanged following OA injection (OA: IL-1 $\beta$ : $t_{17}=0.29 ;$ TNF $\alpha: t_{17}=0.35 ;$ PA: IL- $1 \beta: t_{4}=0.84 ;$ TNF $\alpha$ : $t_{4}=1.25, p>0.05$; Supplementary Figure S1B and C). 

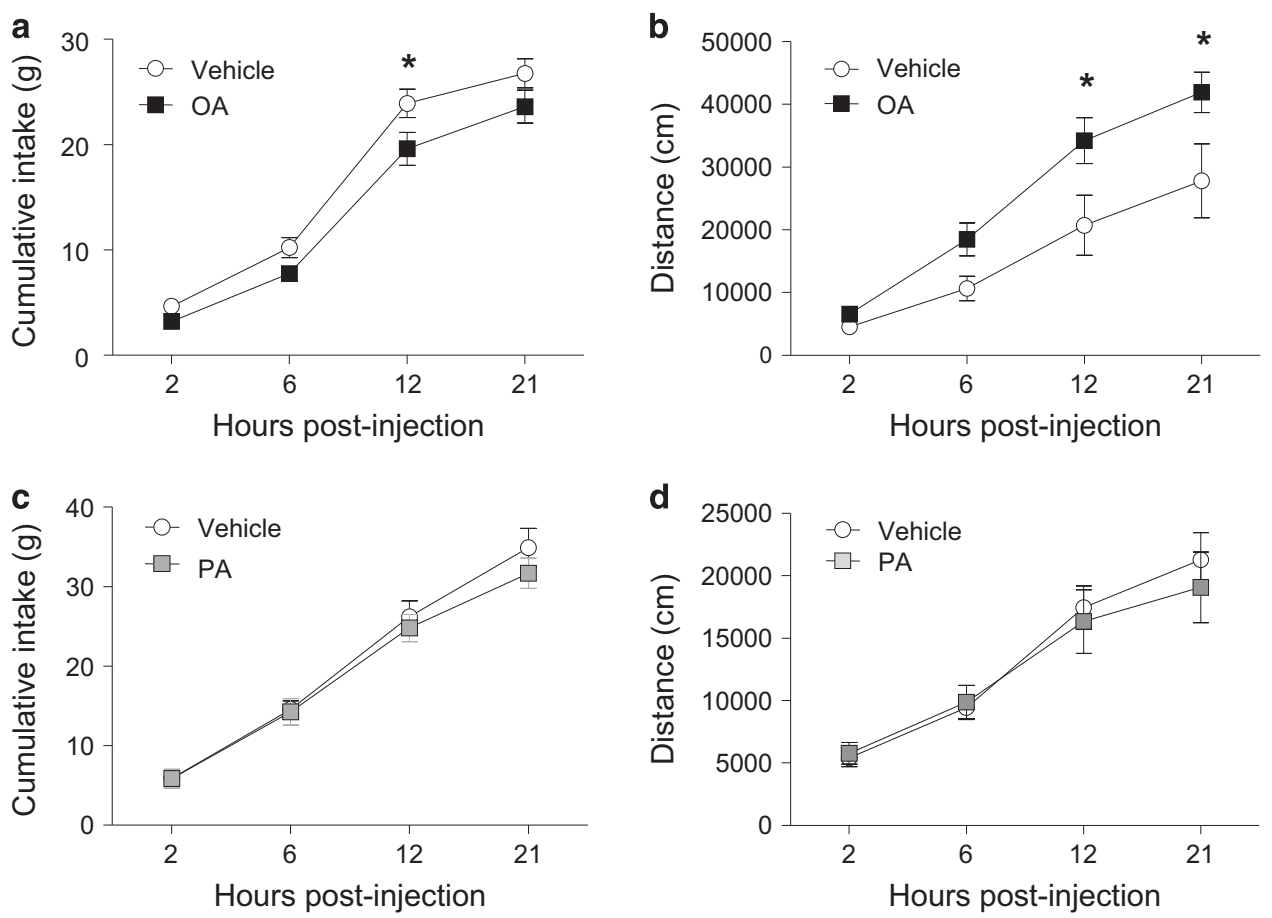

Figure I Intra-VTA oleate inhibits food intake and stimulates locomotor activity. (a, b) Bilateral micro-injection of OA (6 nmol/side) reduced food intake (a) and increased locomotor activity (b). (c, d) Injection of PA into the VTA at an equivalent concentration had no effect on food intake (c) and locomotion (d). $n=8-13 /$ group for food intake assessments; $n=7-10$ /group for locomotor activity experiment. Results are expressed as mean \pm SEM. Two-way repeated measures ANOVA, ${ }^{*} p<0.05$ vs vehicle-injected rats.
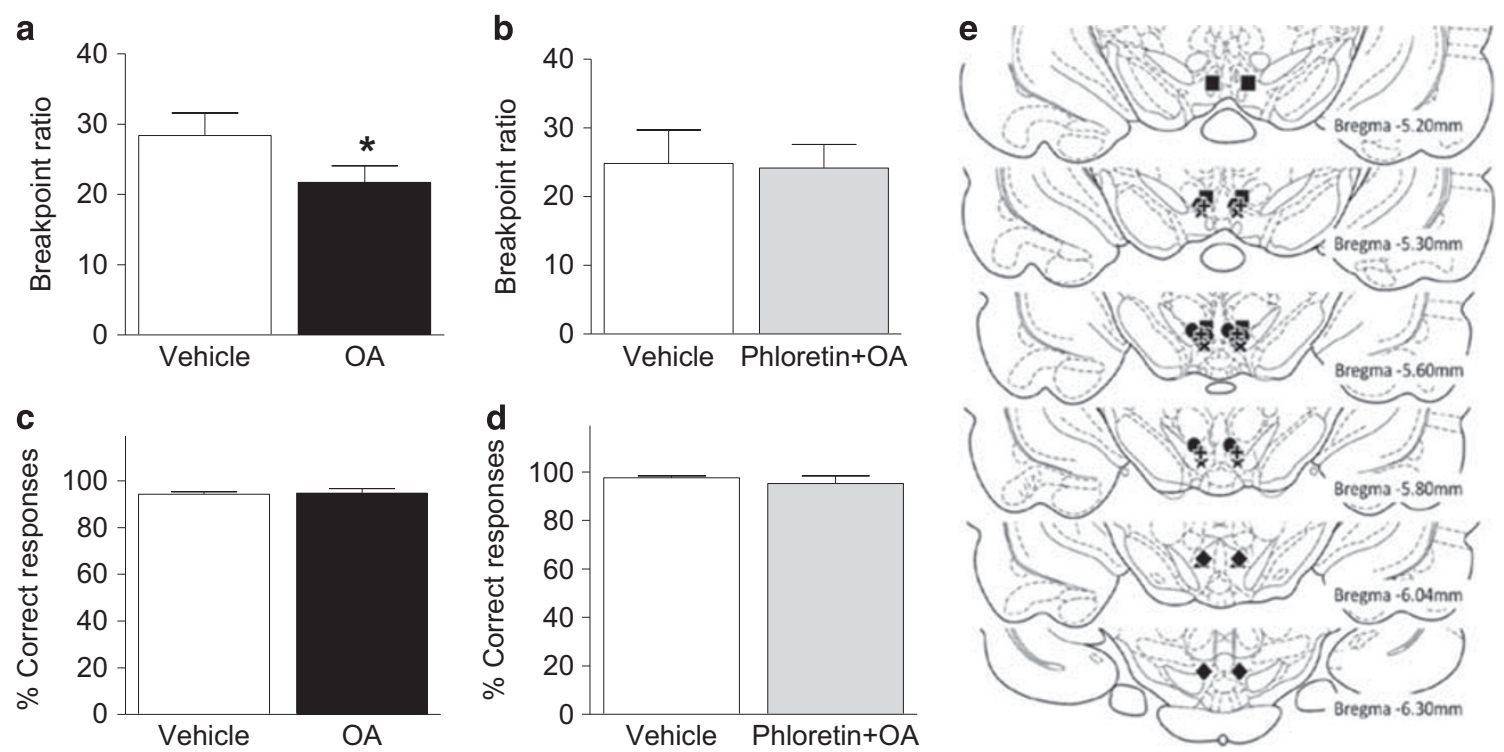

Figure 2 Oleate blunts the rewarding effects of high-fat/-sugar food. (a) Intra-VTA injection of OA decreased breakpoint responding for high-fat/-sucrose pellets in a progressive ratio operant task. (b) The co-injection of the transporter blocker phloretin prevented the effect of OA. (c, d) The percentage of correct lever responses was unaffected by either treatment. (e) Injector cannula placements; each symbol depicts a different rat $(n=7)$. Results are expressed as mean \pm SEM. $* p<0.05$.

\section{Intra-VTA Oleate Suppresses Food Reward}

To determine whether OA alters motivation for palatable food we assessed effort-based, operant responding for highfat/high-sugar food rewards in a progressive ratio task. OA decreased breakpoint thresholds by $23 \%$ relative to vehicle treatment $\left(t_{7}=2.56, p<0.05\right.$; Figure $\left.2 \mathrm{a}\right)$. As the central effects of FA can involve transporter-mediated processes, we next sought to establish whether blocking intracellular uptake could mitigate the ability of OA to suppress foodmotivated behavior. The injection of the transporter blocker phloretin $(100 \mu \mathrm{M} /$ side $)$ alone did not alter chow food intake 
a

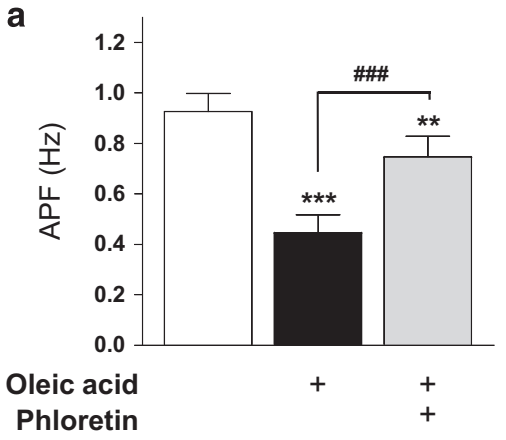

C

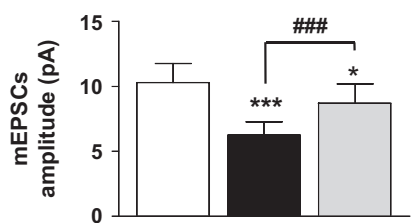

d

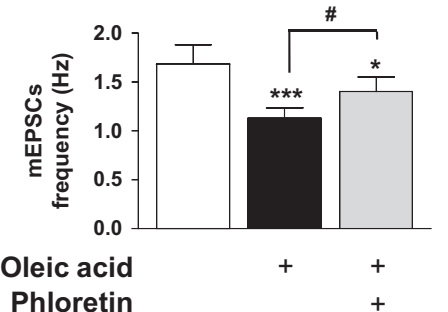

b $\begin{array}{ll}\text { Basal Oleic acid } & \begin{array}{l}\text { Oleic acid } \\ + \text { phloretin }\end{array}\end{array}$

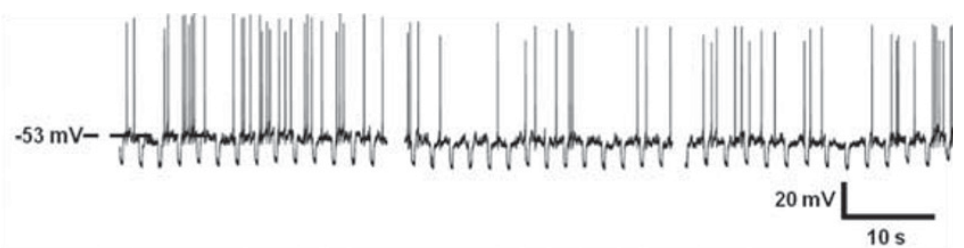

e

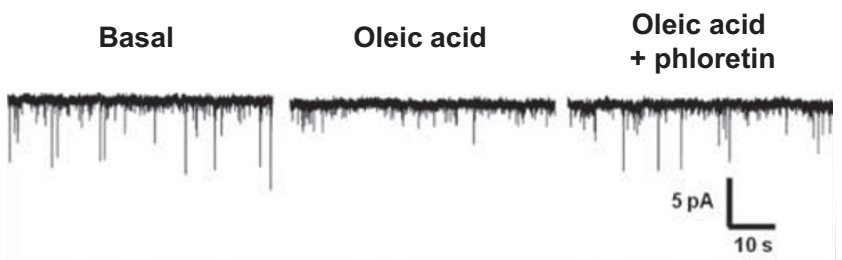

Figure 3 Oleate modulates dopamine neuronal activity. (a) Whole-cell current-clamp recordings of spontaneous electrical activity of VTA DA neurons. OA decreased action potential frequency (APF) of the majority of recorded DA neurons. The co-application of phloretin prevented OA-induced APF inhibition. $n=6$. (b) Representative trace. Resting membrane potential is indicated by the horizontal dashed line and noted on the left. (c, $d$ ) Voltage clamp recordings showing that $O A$ decreased the amplitude $\left(I_{m E P S C}, c\right)$ and the frequency $(d)$ of miniature excitatory post-synaptic currents in OA-inhibited neurons, an effect prevented by phloretin. $n=7$. (e) Representative traces. Downward deflections represent mEPSCs. Results are expressed as mean \pm SEM. One-way standard (a) or repeated measures (c, d) ANOVA. ${ }^{*} p<0.05$; ${ }^{*} * p<0.0$ I; **** $p<0.00$ I vs basal condition; ${ }^{*} p<0.05$; ${ }^{\# \#} p<0.00$ I vs OA condition.

(Supplementary Figure S2; $F_{1,13}=0.59, p>0.05$ ) or high-fat/ high-sugar pellets responding (Supplementary Figure S3; $\left.t_{5}=1.05, p>0.05\right)$. It did however prevent the attenuated breakpoint responding induced by OA $\left(t_{6}=0.31, p>0.05\right.$; Figure $2 \mathrm{~b})$. No changes were observed in the proportion of correct to incorrect lever presses (OA: $t_{7}=0.27$; OA +phloretin: $t_{6}=0.66, p>0.05$; Figure $2 c$ and $\left.d\right)$. Subsequent histological assessment revealed that bilateral cannulas were positioned in the VTA in all rats (Figure 2e).

\section{Oleate Modulates DA Neuronal Activity}

Whole-cell patch recordings were performed to establish whether reductions in food intake and food reward by intraVTA OA are associated with changes in the activity of DA neurons. Current-clamp recordings revealed an effect of OA to inhibit DA neuronal activity: $52 \%$ of neurons $(15 / 29)$ decreased their action potential frequency (APF) in response to OA (basal: $0.91 \pm 0.09 \mathrm{~Hz} v s \mathrm{OA}: 0.44 \pm 0.07 \mathrm{~Hz}$ ). We next ascertained whether OA-induced changes rely on intracellular incorporation of $\mathrm{OA}$ by co-applying phloretin and $\mathrm{OA}$ during current-clamp recordings. Phloretin blocked the effect of OA to inhibit APF (OA: $0.44 \pm 0.07 \mathrm{~Hz} v s$ OA +phloretin: $0.75 \pm 0.08 \mathrm{~Hz} ; \mathrm{F}_{2,14}=8.16, p \leqslant 0.001$; Figure $3 \mathrm{a}$ and $b$, representative trace). We then examined the influence of OA on mEPSCs (in presence of TTX). OA significantly decreased current amplitude (basal: $10.29 \pm 1.48 \mathrm{pA}$ vs OA: $6.25 \pm 1.03 \mathrm{pA}$ ) and frequency (basal: $1.69 \pm 0.19 \mathrm{~Hz} v s \mathrm{OA}:$ $1.13 \pm 0.11 \mathrm{~Hz}$ ), an effect that was partially reversed by coapplication of phloretin (amplitude: OA, $6.25 \pm 1.03 \mathrm{pA} v s$ OA+phloretin, $8.71 \pm 1.49$ pA; frequency: OA, $1.13 \pm 0.11 \mathrm{~Hz}$ vs OA+phloretin, $1.4 \pm 0.15 \mathrm{~Hz}$; amplitude: $\mathrm{F}_{2,6}=44.79$, $p \leqslant 0.0001$; frequency: $\mathrm{F}_{2,6}=25.08, p \leqslant 0.0001$; Figure $3 c-\mathrm{e}$, representative trace). A smaller subset of DA neurons (9/29) exhibited an increase in APF in response to OA (basal: $1.01 \pm 0.28 \mathrm{~Hz}$ vs OA: $1.38 \pm 0.32 \mathrm{~Hz}$ ); however, unlike with OA-inhibited neurons, phloretin had no effect on APF in OA-excited neurons (OA: $1.38 \pm 0.32 \mathrm{~Hz}$ vs OA+phloretin: $\left.1.29 \pm 0.29 \mathrm{~Hz} ; \mathrm{F}_{2,8}=26.04, p<0.0001\right)$. Remaining neurons $(5 / 29)$ were unresponsive to OA (basal: $1.84 \pm 0.74 \mathrm{~Hz}, \mathrm{OA}$ : $\left.1.65 \pm 0.59 \mathrm{~Hz} ; \mathrm{F}_{2,4}=0.55, p>0.05\right)$.

\section{DA Neurons Express FA-Handling Proteins}

Specific isoforms of FA transport proteins (FATP) and FAbinding proteins (FABP) are centrally expressed, namely CD36, FATP1, FATP4, FABP3, FABP5, and FABP7 (Liu et al, 2010; Mitchell et al, 2011b). To determine whether these FA-handling proteins are present in DA neurons we performed qPCR experiments in FACS-purified GFP positive $(\mathrm{GFP}+)$ and negative $(\mathrm{GFP}-)$ cells isolated from the VTA of TH-eGFP mice and compared to expression levels 


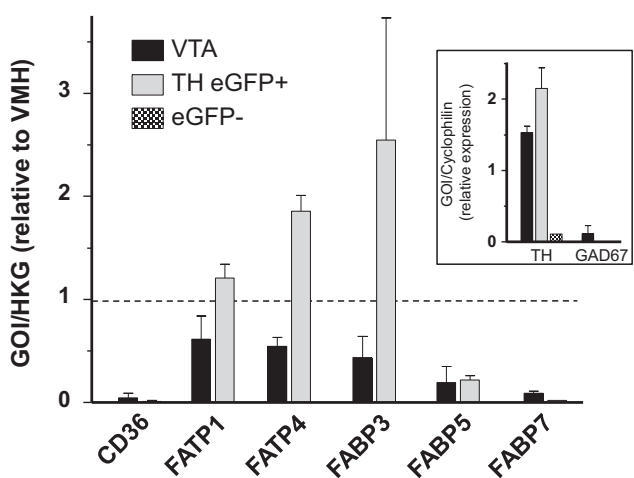

Figure 4 Gene expression profile of fatty acid-handling proteins in dopamine neurons and VTA. DA neurons (GFP+) and non-DA cells (GFP - ) were FACS-sorted from TH-eGFP mice. GFP+ neurons were dopaminergic as evidenced by high levels of TH mRNA and absence of GAD67 mRNA. Cyclophilin served as reference gene and all results (except for TH and GAD67) were normalized to values obtained from $\mathrm{VMH}$ tissue. FABP, fatty acid-binding protein; FATP, fatty acid-transport protein; GAD67, glutamate decarboxylase; TH, tyrosine hydroxylase.

obtained from VTA and VMH microdissections. Given that the metabolic and neurophysiologic actions of FA have been most characterized in the VMH all data were normalized to results obtained from this region. That sorted GFP+ cells were dopaminergic is evidenced by high expression of $T H$ and absence of GAD67 (GABA marker). In contrast, there was minimal $T H$ in GFP - cells. DA neurons and the VTA as a whole were found to express FATP1, FATP4, and FABP3, with minimal expression of $C D 36, F A B P 5$, and $F A B P 7$ (Figure 4).

\section{FAs are Taken Up by DA Neurons}

FA intracellular transport was assessed with BODIPY, a fluorescent long-chain FA analog. BODIPY application to primary DA neurons resulted in punctate labeling in TH immunopositive (TH+) neurons as well as labeling in non$\mathrm{TH}$ cells (Figure $5 \mathrm{e}$, magnified in $5 \mathrm{~h}$ ). Optical $Z$-stack imaging and orthogonal reconstruction revealed cellular BODIPY labeling of spherical form resembling lipid droplets in $\mathrm{TH}+$ cell bodies and processes (Figure $5 \mathrm{e}$ and $\mathrm{f}$ ). The magnitude of BODIPY incorporation was diminished by the addition of phloretin to the incubation medium (Figure $5 \mathrm{f}$ and g). BODIPY application significantly increased cellular fluorescence intensity (expressed as integrated density value) compared to the vehicle condition, whereas co-application of phloretin attenuated the intensity of fluorescent labeling in $\mathrm{TH}+$ neurons $\left(\mathrm{F}_{2,44}=502.0, p \leqslant 0.0001\right.$; Figure $\left.5 \mathrm{j}\right)$.

\section{DISCUSSION}

Central lipid sensing contributes to the control of food intake and energy homeostasis, whereas impediments in this process can dispose to weight gain and obesity. As initial evidence that FA can be detected in the midbrain, the present study reveals that OA suppresses feeding and food reward, and stimulates locomotor activity upon acute administration into the VTA. Shedding light on the cellular mechanisms involved, we demonstrate that OA can inhibit DA neuronal firing, that DA neurons comprise the machinery for FA uptake and shuttling, that long-chain FAs are taken up by DA neurons, and that blocking cellular transport counteracts the behavioral and electrophysiological effects of $\mathrm{OA}$ in the VTA. These findings underscore the capacity of DA neurons to act as metabolic sensors by responding not only to hormones but also to FA nutrient signals to generate adaptive changes in feeding and food-directed behavior.

We chose the intra-VTA study design to determine the effects of FA specifically in the VTA. While it is not yet clear whether circulating FAs reach the VTA we believe it is likely they do given that (1) manipulation of triglyceride hydrolysis into free FAs (knockout of lipoprotein lipase) in extrahypothalamic sites such as the nucleus accumbens (NAc) (Cansell et al, 2014) and hippocampus (Picard et al, 2014b) alters feeding behavior; (2) lipoprotein lipase is enriched in the VTA, at concentrations that are higher than other brain regions (Chung et al, 2005; Greene et al, 2005; Grimm et al, 2004); (3) as demonstrated in the present work, FA transporters and binding proteins are expressed in the VTA, in dopaminergic and non-dopaminergic cells; and (4) multiple metabolic signals (insulin, leptin, ghrelin, GLP-1, and amylin) that target the hypothalamus also have signaling actions in the VTA. While the physiological levels of FA in the VTA are unknown, the $6 \mathrm{nmol}$ dose was extrapolated from a combination of previous reports (Obici et al, 2002; Ross et al, 2010; Schwinkendorf et al, 2011) and our own experiments testing ICV oleate on food intake. Despite a more physiological route to the VTA, intravenous or intragastric administration would not permit establishing whether FAs are detected by the VTA to modulate behavior. Indeed, an intra-gastric infusion of FA would modulate secretion of various incretin hormones such as GLP-1, stimulate the enteric nervous system, and modulate oleoylethanolamides, each of which can affect the mesolimbic DA system (Fortin et al, 2016; Tellez et al, 2013). Similarly, an i.v. infusion of FA would stimulate insulin secretion, which is also known to target VTA DA neurons (Labouebe et al, 2013). The anorectic influence of intra-VTA OA observed is consistent with its actions to decrease food intake when delivered into the third ventricle (Obici et al, 2002; Schwinkendorf et al, 2011). Moreover, our results distinguish the impact of OA by demonstrating that the same dose of PA, another ubiquitous long-chain FA, fails to affect food consumption when administered into the VTA. We cannot exclude, however, that a higher concentration of PA might affect feeding. Importantly, the infusion of either FA was not associated with local toxicity and inflammation. These findings are in line with previous evidence that unsaturated FA species (OA and docosahexanoic acid), but not saturated FA like PA, have an anorectic action upon intraventricular infusion (Schwinkendorf et al, 2011). PA has been more associated with inflammatory pathway stimulation and diminished central leptin and insulin signaling (Benoit et al, 2009; Kleinridders et al, 2009). Concomitant with reduced feeding, rats exhibited an increase in locomotor activity following OA (but not PA) injection, an effect that could be due to increased time available for behaviors other than feeding and/or to direct cellular actions of OA in the VTA. Although others have reported opposite effects of OA on locomotion (Contreras et al, 2014; Gobbi et al, 1999), the peripheral 

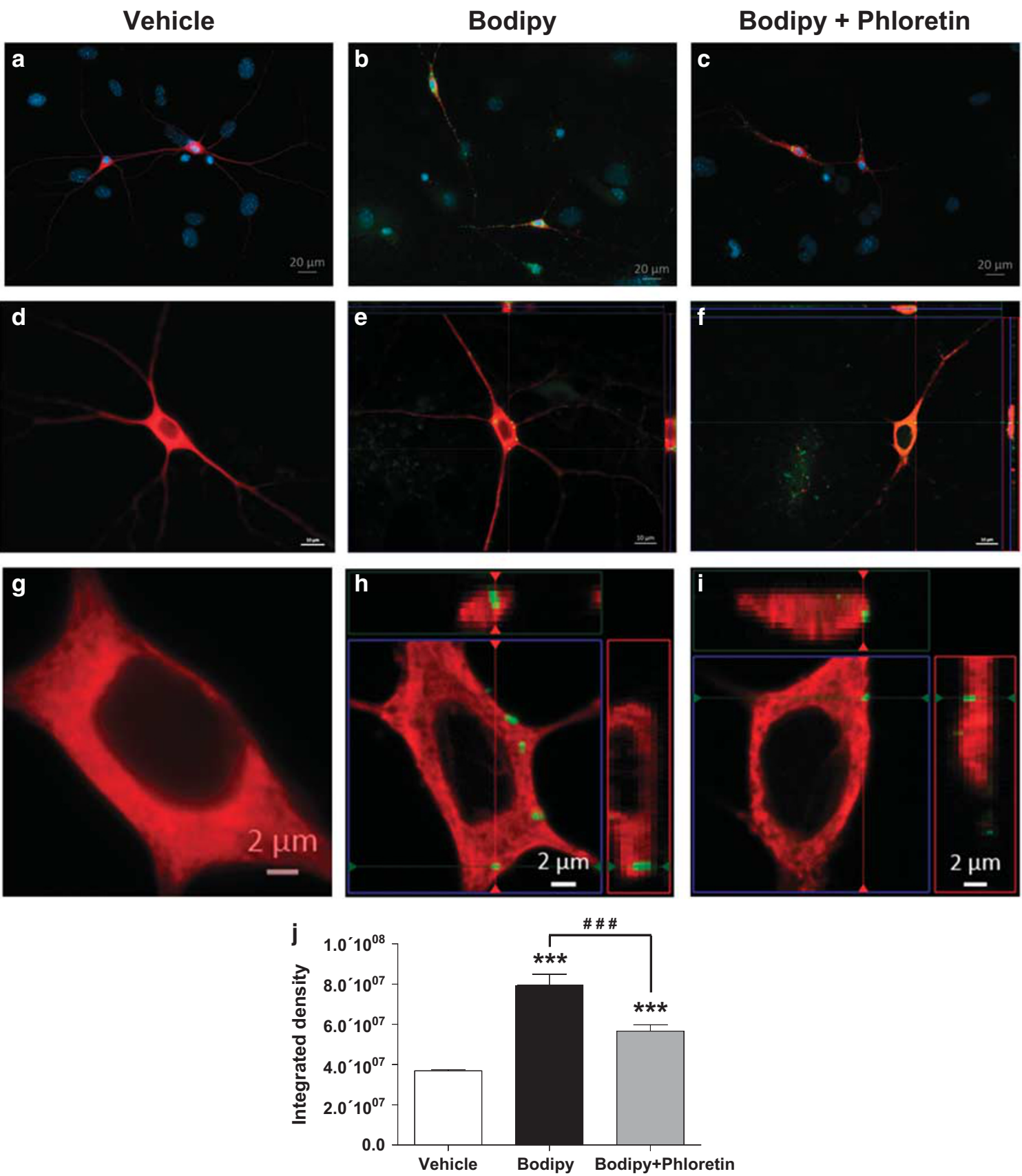

Figure 5 Fatty acid uptake in primary dopamine neurons. Application of the long-chain FA analog BODIPY to the culture medium resulted in intracellular punctuate labeling (green) in the cytoplasm as well as processes of DA neurons identified by TH immunofluorescence (red). (a, $d, g)$ Vehicle application. (b, e, h) BODIPY $(20 \mu \mathrm{M})$ application. (c, f, i) BODIPY $(20 \mu \mathrm{M})$ +phloretin (I00 $\mu \mathrm{M})$ application. (j) BODIPY fluorescence intensity values across treatment conditions. Three coverslips per condition. Results are expressed as mean \pm SEM. One-way ANOVA. **** $p<0.00$ I vs vehicle; $\# \# p<0.00$ I vs BODIPY alone. $\mathrm{TH}$, tyrosine hydroxylase.

route of administration in these studies may well explain the discrepancy with our results.

As the VTA and its corticolimbic targets are a critical component of the circuitry controlling motivation, we investigated the effect of $\mathrm{OA}$ on effort-based responding for palatable food. Acute OA into the VTA diminished the rewarding effects of high-fat/-sucrose food. This finding is in agreement with previous work demonstrating that intracarotid infusions of a lipid emulsion (intralipid-comprising mostly polyunsaturated and monounsaturated FAs) reduces food-motivated responding (Cansell et al, 2014). Moreover, Cansell and colleagues demonstrated that deletion of the gene encoding lipoprotein lipase, an enzyme hydrolyzing FA from triacylglycerol, from the NAc blocks the effects of lipid infusion to decrease food reward. It remains to be determined whether the effect of moderating FA levels in the NAc on food-seeking is mediated by distinct FA such as $\mathrm{OA}$ and which mechanisms are involved.

In accordance with blunted motivation and food seeking, the firing frequency of the majority of recorded DA neurons 
was reduced in response to OA application. As has been demonstrated with other anorectic hormones and peptides (Liu and Borgland, 2015), OA can directly inhibit DA neuronal activity thereby bypassing FA-sensing mechanisms of the hypothalamus. Similar to observations in the hypothalamus (Jo et al, 2009; Wang et al, 2006), 80\% of DA neurons responded to OA, 52\% were inhibited, and 29\% were excited by OA. While this possibility remains to be tested, DA neurons excited by OA could be involved in the locomotor-enhancing effect of intra-VTA OA injection. As we previously showed, a subset of leptin-responsive DA neurons in the VTA modulates locomotor activity and running reward (Fernandes et al, 2015) and thus perhaps oleate may have similar actions by stimulating the activity of these neurons. These divergent neuronal activity changes in response to OA suggest that DA neurons can be distinguished based on their response to nutritional signals. Indeed, heterogeneity in the functional, anatomic, electrophysiological, and genetic properties of VTA DA neurons is well documented (Morales and Margolis, 2017). In this regard it is important to mention that as we used rats and mice for our experiments, differences in the activity of VTA DA neurons across species may limit the interpretation of our findings. OA treatment had no effect on membrane potential or input resistance suggesting that it does not modulate non-synaptic (background or leak) ion channel closure/opening. OA significantly reduced the frequency of mEPSCs indicating a reduction in the probability of presynaptic glutamate release. The decrease in mEPSC amplitude may reflect this decrease in transmitter release; however, it could also suggest post-synaptic modifications of ionotropic glutamate receptors.

FATPs and FABPs are centrally expressed and contribute to cell differentiation, FA uptake, energy balance, and cognitive processes (Moulle et al, 2012). While there is de novo FA synthesis in the brain (Medina and Tabernero, 2002), the FAs incorporated in neural cells are largely derived from the periphery. Our results demonstrate that DA neurons express the machinery for intracellular FA transport and shuttling, and that they are capable of FA incorporation. Interestingly, expression of CD36, the FATP most tied to the effects of intra-hypothalamic FA on energy balance (Le Foll et al, 2013; Moulle et al, 2013), was minimal or absent in the VTA and in purified DA neurons, respectively. Rather, FATP1 and FATP4 were expressed at relatively elevated levels and thus could participate in cellular uptake and behavioral actions of OA in VTA. Among FA-binding proteins expressed in brain, FABP5 is predominantly expressed in immature neurons and glia, FABP7 in glial cells, and FABP3 is enriched in mature neurons (Mitchell and Hatch, 2011a). Correspondingly, DA neurons exhibited high levels of FABP3 (compared to whole VTA and VMH) and minimal FABP5 and FABP7 expression, a finding consistent with reports of FABP3 expression in substantia nigra DA neurons in mice (Shioda et al, 2014). FABP3 also exhibits considerable affinity for monounsaturated FA such as OA (Tan et al, 2014) to further suggest a potential role for FABP3 in mediating the effect of OA in DA neurons. Along with the expression of genes encoding FA-handling proteins we found that DA neurons are capable of intracellular FA transport. While BODIPY FA is not a perfect analog for oleate, it is processed by cells as a long-chain FA (Carten et $a l, 2011$ ) and is readily taken up by neurons (Kaushik et al, 2011). BODIPY accumulated in structures resembling lipid droplets, which are intracellular organelles that store triglycerides. This observation is consistent with our previous work showing that $\mathrm{OA}$ is esterified into triglycerides in hypothalamic neurons (Taib et al, 2013) and suggests that the metabolic fate of FA is similar between VTA DA and hypothalamic neurons.

The mechanisms by which FA gain entry into cells are not entirely clear, however several lines of evidence implicate active transporter-mediated processes (Mitchell and Hatch, 2011a). With the application of phloretin, a nonspecific inhibitor of FA transport (Chabowski et al, 2013), we show that blocking transport diminishes FA uptake into DA neurons and prevents OA-induced suppression of foodmotivated responding and DA neuronal activity. Along with the gene expression profile, these results suggest that the behavioral and physiological actions of intra-VTA OA at least partly rely on interactions with transporters and/or binding proteins. In the hypothalamus, the effects of FA on neuronal activity and feeding are largely tied to the membrane FA transporter CD36 and are shown to be independent of FA intracellular metabolism (Le Foll et al, 2013; Moulle et al, 2013). However, FA-induced alterations in ion channel activity as well as FA mitochondrial oxidation can also account for the cellular effects of FA (reviewed in Picard et al, 2014a). Such alternative pathways may be involved in the actions of OA observed here as CD36 expression was barely detectable in DA neurons and VTA. Although our FA uptake and electrophysiological data support a direct effect of OA on DA neurons, we cannot exclude the possibility that OA modulates DA neurons via regulation of GABA inputs or neighboring astrocytes. Indeed, astrocytes take up, metabolize, and release FA (Bouyakdan et al, 2015; Taib et al, 2013) and FA oxidation in astrocytes produces ketone bodies (KBs) (Bixel and Hamprecht, 1995) that could in turn alter neuronal activity (Guzman and Blazquez, 2001; Ma et al, 2007). Moreover, Le Foll et al (2014) describe that hypothalamic KB generation induced by high-fat feeding decreases food intake. Thus, we cannot rule out the contribution of FA-derived $\mathrm{KB}$ and look toward future investigations to identify the metabolic and molecular signals involved in the impact of OA in the VTA.

\section{CONCLUSION}

The collective results suggest that OA in the VTA represents a signal of energy surfeit to curb food intake and foodoriented behaviors. That these catabolic actions were selective to a monounsaturated but not saturated longchain FA further emphasizes the significance of FA type in the central signaling and metabolic action of FA. The effects of OA to suppress appetite and food-motivated behavior were linked to OA-induced inhibition of DA neuronal firing and to interactions with FA transporters, possibly including intracellular OA uptake. Brain FA content is fairly stable in response to short-term dietary changes in FA; however, human obesity and the metabolic syndrome are linked to increased central FA uptake (Karmi et al, 2010). Thus, a question for future research is whether VTA sensing of FA is altered by prolonged consumption of energy-dense food and 
the development of obesity, and whether such changes could contribute to uncontrolled reward-seeking and/or hyperphagia and weight gain.

\section{FUNDING AND DISCLOSURE}

The authors declare no conflict of interest.

\section{ACKNOWLEDGMENTS}

We thank Marie-Josée Bourque for neuron cultures and help with cell sorting, and Demetra Rodaros and Anna Kristyna Franco Flores for helping with intra-VTA surgeries and operant responding experiments. This work was supported by a CIHR grant (MOP123280) and New Investigator salary award to SF, by a CIHR grant (MOP115042) and Fonds de Recherche Québec-Santé salary award to TA, by CIHR (MOP106556) and Brain Canada/Krembil Foundation grants to L-ET, American Heart Association grants to VHR (14GRNT20380639 and 1RO1DK103676), and doctoral scholarships from the Montreal Diabetes Research Center/ Université de Montréal and CMDO/Novo Nordisk to $\mathrm{CH}$.

\section{REFERENCES}

Benoit SC, Kemp CJ, Elias CF, Abplanalp W, Herman JP, Migrenne $S$ et al (2009). Palmitic acid mediates hypothalamic insulin resistance by altering PKC-theta subcellular localization in rodents. J Clin Invest 119: 2577-2589.

Bixel MG, Hamprecht B (1995). Generation of ketone bodies from leucine by cultured astroglial cells. J Neurochem 65: 2450-2461.

Bouyakdan K, Taib B, Budry L, Zhao S, Rodaros D, Neess D et al (2015). A novel role for central ACBP/DBI as a regulator of longchain fatty acid metabolism in astrocytes. J Neurochem 133: 253-265.

Cansell C, Castel J, Denis RG, Rouch C, Delbes AS, Martinez S et al (2014). Dietary triglycerides act on mesolimbic structures to regulate the rewarding and motivational aspects of feeding. $\mathrm{Mol}$ Psychiatry 19: 1095-1105.

Carten JD, Bradford MK, Farber SA (2011). Visualizing digestive organ morphology and function using differential fatty acid metabolism in live zebrafish. Dev Biol 360: 276-285.

Chabowski A, Żendzian-Piotrowska M, Konstantynowicz K, Pankiewicz W, Mikłosz A, Łukaszuk B et al (2013). Fatty acid transporters involved in the palmitate and oleate induced insulin resistance in primary rat hepatocytes. Acta Physiol 207: 346-357.

Chung CY, Seo H, Sonntag KC, Brooks A, Lin L, Isacson O (2005). Cell type-specific gene expression of midbrain dopaminergic neurons reveals molecules involved in their vulnerability and protection. Hum Mol Genet 14: 1709-1725.

Contreras CM, Rodriguez-Landa JF, Garcia-Rios RI, CuetoEscobedo J, Guillen-Ruiz G, Bernal-Morales B (2014). Myristic acid produces anxiolytic-like effects in Wistar rats in the elevated plus maze. BioMed Res Int 2014: 492141.

Davis JF, Tracy AL, Schurdak JD, Tschop MH, Lipton JW, Clegg DJ et al (2008). Exposure to elevated levels of dietary fat attenuates psychostimulant reward and mesolimbic dopamine turnover in the rat. Behav Neurosci 122: 1257-1263.

Décarie-Spain L, Hryhorczuk C, Fulton S (2016). Dopamine signalling adaptations by prolonged high-fat feeding. Curr Opin Behav Sci 9: 136-143.

Fasano C, Thibault D, Trudeau LE (2008). Culture of postnatal mesencephalic dopamine neurons on an astrocyte monolayer. Curr Protoc Neurosci Chapter 3: Unit 3.21.
Fernandes MF, Matthys D, Hryhorczuk C, Sharma S, Mogra S, Alquier $\mathrm{T}$ et al (2015). Leptin suppresses the rewarding effects of running via STAT3 signaling in dopamine neurons. Cell Metab 22: 741-749.

Fortin SM, Chartoff EH, Roitman MF (2016). The aversive agent lithium chloride suppresses phasic dopamine release through central GLP-1 receptors. Neuropsychopharmacology 41: 906-915.

Fulton S (2010). Appetite and reward. Front Neuroendocrinol 31: 85-103.

Fulton S, Pissios P, Manchon RP, Stiles L, Frank L, Pothos EN et al (2006). Leptin regulation of the mesoaccumbens dopamine pathway. Neuron 51: 811-822.

Fulton S, Thibault D, Mendez JA, Lahaie N, Tirotta E, Borrelli E et al (2011). Contribution of Kv1.2 voltage-gated potassium channel to D2 autoreceptor regulation of axonal dopamine overflow. J Biol Chem 286: 9360-9372.

Gobbi M, Mennini T, Dalla Valle F, Cervo L, Salmona M, Diomede L (1999). Oleamide-mediated sleep induction does not depend on perturbation of membrane homeoviscosity. FEBS Lett 463: 281-284.

Greene JG, Dingledine R, Greenamyre JT (2005). Gene expression profiling of rat midbrain dopamine neurons: implications for selective vulnerability in parkinsonism. Neurobiol Dis 18: 19-31.

Grimm J, Mueller A, Hefti F, Rosenthal A (2004). Molecular basis for catecholaminergic neuron diversity. Proc Natl Acad Sci USA 101: 13891-13896.

Guzman M, Blazquez C (2001). Is there an astrocyte-neuron ketone body shuttle? Trends Endocrinol Metab 12: 169-173.

Hryhorczuk C, Florea M, Rodaros D, Poirier I, Daneault C, Des Rosiers C et al (2015). Dampened mesolimbic dopamine function and signaling by saturated but not monounsaturated dietary lipids. Neuropsychopharmacology 41: 811-821.

Jo YH, Su Y, Gutierrez-Juarez R, Chua S Jr. (2009). Oleic acid directly regulates POMC neuron excitability in the hypothalamus. J Neurophysiol 101: 2305-2316.

Johnson PM, Kenny PJ (2010). Dopamine D2 receptors in addiction-like reward dysfunction and compulsive eating in obese rats. Nat Neurosci 13: 635-641.

Karmi A, Iozzo P, Viljanen A, Hirvonen J, Fielding BA, Virtanen K et al (2010). Increased brain fatty acid uptake in metabolic syndrome. Diabetes 59: 2171-2177.

Kaushik S, Rodriguez-Navarro JA, Arias E, Kiffin R, Sahu S, Schwartz GJ et al (2011). Autophagy in hypothalamic AgRP neurons regulates food intake and energy balance. Cell Metab 14: 173-183.

Kleinridders A, Schenten D, Konner AC, Belgardt BF, Mauer J, Okamura T et al (2009). MyD88 signaling in the CNS is required for development of fatty acid-induced leptin resistance and dietinduced obesity. Cell Metab 10: 249-259.

Labouebe G, Liu S, Dias C, Zou H, Wong JC, Karunakaran S et al (2013). Insulin induces long-term depression of ventral tegmental area dopamine neurons via endocannabinoids. Nat Neurosci 16: 300-308.

Lam TK, Pocai A, Gutierrez-Juarez R, Obici S, Bryan J, AguilarBryan L et al (2005). Hypothalamic sensing of circulating fatty acids is required for glucose homeostasis. Nat Med 11: 320-327.

Le Foll C, Dunn-Meynell A, Musatov S, Magnan C, Levin BE (2013). FAT/CD36: a major regulator of neuronal fatty acid sensing and energy homeostasis in rats and mice. Diabetes 62: 2709-2716.

Le Foll C, Dunn-Meynell AA, Miziorko HM, Levin BE (2014). Regulation of hypothalamic neuronal sensing and food intake by ketone bodies and fatty acids. Diabetes 63: 1259-1269.

Le Foll C, Irani BG, Magnan C, Dunn-Meynell AA, Levin BE (2009). Characteristics and mechanisms of hypothalamic neuronal fatty acid sensing. Am J Physiol Regul Integr Comp Physiol 297: R655-R664.

Liu RZ, Mita R, Beaulieu M, Gao Z, Godbout R (2010). Fatty acid binding proteins in brain development and disease. Int J Dev Biol 54: 1229-1239. 
Liu S, Borgland SL (2015). Regulation of the mesolimbic dopamine circuit by feeding peptides. Neuroscience 289: 19-42.

Ma W, Berg J, Yellen G (2007). Ketogenic diet metabolites reduce firing in central neurons by opening K(ATP) channels. J Neurosci 27: 3618-3625.

Matsushita N, Okada H, Yasoshima Y, Takahashi K, Kiuchi K, Kobayashi K (2002). Dynamics of tyrosine hydroxylase promoter activity during midbrain dopaminergic neuron development. $J$ Neurochem 82: 295-304.

Medina JM, Tabernero A (2002). Astrocyte-synthesized oleic acid behaves as a neurotrophic factor for neurons. J Physiol Paris 96: 265-271.

Mendez JA, Bourque M-J, Bo GD, Bourdeau ML, Danik M, Williams $S$ et al (2008). Developmental and target-dependent regulation of vesicular glutamate transporter expression by dopamine neurons. J Neurosci 28: 6309-6318.

Mitchell RW, Hatch GM (2011a). Fatty acid transport into the brain: of fatty acid fables and lipid tails. Prostaglandins Leukot Essent Fatty Acids 85: 293-302.

Mitchell RW, On NH, Del Bigio MR, Miller DW, Hatch GM (2011b). Fatty acid transport protein expression in human brain and potential role in fatty acid transport across human brain microvessel endothelial cells. J Neurochem 117: 735-746.

Morales M, Margolis EB (2017). Ventral tegmental area: cellular heterogeneity, connectivity and behaviour. Nat Rev Neurosci 18: 73-85.

Moulle VS, Cansell C, Luquet S, Cruciani-Guglielmacci C (2012). The multiple roles of fatty acid handling proteins in brain. Front Physiol 3: 385.

Moulle VS, Le Foll C, Philippe E, Kassis N, Rouch C, Marsollier N et al (2013). Fatty acid transporter CD36 mediates hypothalamic effect of fatty acids on food intake in rats. PLOS ONE 8: e74021.

Obici S, Feng Z, Arduini A, Conti R, Rossetti L (2003). Inhibition of hypothalamic carnitine palmitoyltransferase-1 decreases food intake and glucose production. Nat Med 9: 756-761.

Obici S, Feng Z, Morgan K, Stein D, Karkanias G, Rossetti L (2002). Central administration of oleic acid inhibits glucose production and food intake. Diabetes 51: 271-275.

Oomura Y, Nakamura T, Sugimori M, Yamada Y (1975). Effect of free fatty acid on the rat lateral hypothalamic neurons. Physiol Behav 14: 483-486.

Paxinos G, Watson C. The Rat Brain in Stereotaxic Coordinates. Academic Press: Cambridge, MA, 1998.

Picard A, Moulle VS, Le Foll C, Cansell C, Veret J, Coant N et al (2014a). Physiological and pathophysiological implications of lipid sensing in the brain. Diabetes Obes Metab 16(Suppl 1): 49-55.
Picard A, Rouch C, Kassis N, Moulle VS, Croizier S, Denis RG et al (2014b). Hippocampal lipoprotein lipase regulates energy balance in rodents. Mol Metab 3: 167-176.

Ross RA, Rossetti L, Lam TK, Schwartz GJ (2010). Differential effects of hypothalamic long-chain fatty acid infusions on suppression of hepatic glucose production. Am J Physiol Endocrinol Metab 299: E633-E639.

Schwinkendorf DR, Tsatsos NG, Gosnell BA, Mashek DG (2011). Effects of central administration of distinct fatty acids on hypothalamic neuropeptide expression and energy metabolism. Int J Obes (Lond) 35: 336-344.

Sharma S, Hryhorczuk C, Fulton S (2012). Progressive-ratio responding for palatable high-fat and high-sugar food in mice. $J$ Vis Exp 63: e3754.

Sheng Z, Santiago AM, Thomas MP, Routh VH (2014). Metabolic regulation of lateral hypothalamic glucose-inhibited orexin neurons may influence midbrain reward neurocircuitry. Mol Cell Neurosci 62: 30-41.

Shioda N, Yabuki Y, Kobayashi Y, Onozato M, Owada Y, Fukunaga $\mathrm{K}$ (2014). FABP3 protein promotes alpha-synuclein oligomerization associated with 1-methyl-1,2,3,6-tetrahydropiridine-induced neurotoxicity. J Biol Chem 289: 18957-18965.

Stice E, Spoor S, Bohon C, Veldhuizen MG, Small DM (2008). Relation of reward from food intake and anticipated food intake to obesity: a functional magnetic resonance imaging study. $J$ Abnorm Psychol 117: 924-935.

Taib B, Bouyakdan K, Hryhorczuk C, Rodaros D, Fulton S, Alquier T (2013). Glucose regulates hypothalamic long-chain fatty acid metabolism via AMP-activated kinase (AMPK) in neurons and astrocytes. J Biol Chem 288: 37216-37229.

Tan MC, Matsuoka S, Ano H, Ishida H, Hirose M, Sato F et al (2014). Interaction kinetics of liposome-incorporated unsaturated fatty acids with fatty acid-binding protein 3 by surface plasmon resonance. Bioorg Med Chem 22: 1804-1808.

Tellez LA, Medina S, Han W, Ferreira JG, Licona-Limon P, Ren X et al (2013). A gut lipid messenger links excess dietary fat to dopamine deficiency. Science (New York, NY) 341: 800-802.

Wang GJ, Volkow ND, Logan J, Pappas NR, Wong CT, Zhu W et al (2001). Brain dopamine and obesity. Lancet 357: 354-357.

Wang R, Cruciani-Guglielmacci C, Migrenne S, Magnan C, Cotero VE, Routh VH (2006). Effects of oleic acid on distinct populations of neurons in the hypothalamic arcuate nucleus are dependent on extracellular glucose levels. J Neurophysiol 95: 1491-1498.

Supplementary Information accompanies the paper on the Neuropsychopharmacology website (http://www.nature.com/npp) 